\title{
Precise destruction: an emerging picture of the APC
}

\author{
Brian R. Thornton and David P. Toczyski ${ }^{1}$ \\ Department of Biochemistry and Biophysics, Cancer Research Institute, University of California at San Francisco, \\ San Francisco, California 94115, USA
}

Cell cycle transitions are often accompanied by the degradation of regulatory molecules. Targeting proteins to the proteasome for degradation is accomplished by the covalent addition of ubiquitin chains. The specificity of this pathway is largely dictated by a set of enzymes called ubiquitin ligases (or E3s). The anaphase-promoting complex (or APC) is a ubiquitin ligase that has a particularly prominent role in regulating cell cycle progression. To date, the APC is the most complicated member of the RING/cullin family of multisubunit E3s. It includes at least 13 core subunits and three related adaptors. A combination of biochemical, genetic, and structural approaches are now shedding light on the enzymology of the APC. This review will focus on these data, drawing parallels with related ubiquitin ligases.

\section{Ubiquitin ligases and the cell cycle}

Proteolysis is a particularly powerful tool in the cellular regulatory toolbox, most clearly so in the process of eukaryotic cell division. The driving force behind the cell division cycle is a system of tightly regulated oscillating waves of cyclin-dependent kinase (CDK) activity. Whereas higher organisms use several CDK kinase subunits, budding and fission yeast each have a single CDK that promotes cell cycle progression, called Cdc28 and $\mathrm{Cdc} 2$, respectively. We will refer to all of these collectively as CDK unless stated otherwise. By pairing with different cyclin subunits, CDKs alternately drive or inhibit crucial cell division events, allowing processes such as DNA replication, chromosome condensation, spindle assembly and disassembly, nuclear division, and cytokinesis to occur in the proper order and with proper timing (Murray 2004). These oscillations in CDK activity are accomplished in large part due to the action of ubiquitin ligases: either directly, by controlling the levels of cyclins, or indirectly, by controlling the levels of CDK inhibitors. Proteolysis is particularly useful for driving such oscillations because it is effectively irreversible, requiring new protein synthesis each cycle and thus forcing directionality on the system. However, it is

[Keywords: APC; Cdc20; ubiquitin; yeast]

${ }^{1}$ Corresponding author.

E-MAIL toczyski@cc.ucsf.edu; FAX (415) 502-3179.

Article is online at http://www.genesdev.org/cgi/doi/10.1101/gad.1478306. also a costly and potentially dangerous process that must be tightly regulated.

Ubiquitin ligases (sometimes called "E3s") transfer ubiquitin that has previously been covalently attached to a ubiquitin-conjugating enzyme (an "E2"), which in turn receives its ubiquitin from a ubiquitin-activating enzyme, or E1. Most ubiquitin ligases fall into either of two main groups, depending on the class of catalytic subunit they employ (Pickart 2001). One group uses HECT domain-containing proteins to mediate the ubiquitin linkage. These proteins generate a covalent thioester linkage with ubiquitin before attaching it to a substrate. Another class of ubiquitin ligases contains a Zn-coordinating RING finger. RING finger ubiquitin ligases are not thought to generate a covalent linkage with ubiquitin, but instead direct the transfer of ubiquitin from the E2 to the substrate. For both of these classes of E3, the transfer of ubiquitin to the substrate is ATP-independent. Beyond the conserved RING domain, RING finger ligases are enormously heterogenous. While several act as single-subunit enzymes, others are parts of large multiprotein complexes. Several members of this latter group contain a member of the cullin family of proteins and a set of loosely associated adaptor proteins that serve in part to recognize substrates. Two of the best-studied members of this subclass are the APC / the anaphasepromoting complex or cyclosome) and the SCF (Skp/Cullin/F-box).

The APC was initially characterized for its ability to target the vertebrate and yeast mitotic cyclins for degradation (Irniger et al. 1995; King et al. 1995; Sudakin et al. 1995; Tugendreich et al. 1995), an essential task since mitotic cyclin/CDK activity inhibits the licensing of origins for new rounds of DNA synthesis, cytokinesis, and exit from mitosis (Shirayama et al. 1999; Wasch and Cross 2002). The second key target of the APC in yeast and mammals is securin, a protein that blocks the metaphase-to-anaphase transition by binding to and inhibiting the protease activity of separase (Cohen-Fix et al. 1996; Shirayama et al. 1999). The APC also targets many other proteins for degradation, including the yeast motor proteins Cin8 and Kip1, the yeast spindle protein Ase1, mitotic regulators such as the yeast and mammalian polo kinases, regulators of DNA replication such as Dbf4 in yeast and geminin in mammals, and the APC adaptor 
protein Cdc20 (for review, see Reed 2003). Proteins targeted by the APC are often targeted by it in all eukaryotes examined; however, there are exceptions. For example, the regulator of replication initiation Cdc6 is targeted by the SCF in yeast and the APC in mammals. While the targeting of several of these proteins is critical for cell cycle regulation, the degradation of others is largely redundant with other forms of regulation. In yeast, it has been shown that of these APC targets, only securin and the mitotic cyclins must be degraded for cells to live (Thornton and Toczyski 2003).

The two largest obstacles to understanding APC structure and mechanism are the lack of an APC crystal structure and our inability to reconstitute the APC from purified subunits. The APC is a large enzyme, composed of at least 13 core subunits (see Table 1), making study of the complex as a whole difficult. The development of a robust in vitro assay using APC purified from yeast has allowed investigators to examine the activity of endogenous, wild-type APC and APC purified from genetically altered strains (Carroll and Morgan 2005; Herzog and Peters 2005). The identification of conditions that render the APC nonessential in yeast (Thornton and Toczyski 2003) has dramatically increased our ability to manipulate endogenously purified APC. This has allowed the creation of a complete subunit assembly map (Fig. 1A), and allowed in vitro ubiquitination and adaptor-binding assays to be performed on APC subcomplexes (or mutant complexes) that do not normally support viability (Thornton et al. 2006). Additionally, structural information is now available through recent APC EM structures (Fig. 1B-D; Gieffers et al. 2001; Dube et al. 2005; Pass- more et al. 2005). While they do not provide atomic resolution, EM studies have the advantage of providing structural data at the single molecule/complex level. This has generated data on multiple forms of these structures (to be discussed): Barford and colleagues (Passmore et al. 2005) identified both a monomeric and dimeric form of the APC; Stark and colleagues (Dube et al. 2005) found that two forms of the monomeric APC could be identified that differed in the angle of one large domain, suggesting flexibility within the complex (Fig. 1D). While EM does not provide the resolution to allow explicit subunit identification, immuno-EM was used to identify some subunits within these structures.

An emerging view of the APC is that of a four-part enzyme, composed of a structural complex (Apc1/Apc4/ Apc5), a catalytic arm that houses the E2-binding sites (Apc2/Apc11/Doc1), a tetratricopeptide repeat (TPR) arm involved primarily in adaptor binding (Cdc23/Cdc16/ Cdc27/Cdc26/Swm1), and the adaptors that help bridge the interaction with substrates (Cdc20/Cdh1/Ama1). The APC without the associated adaptors is referred to as the "APC core." We will discuss recent advances in our understanding of the TPR and Cullin arms of the APC, as well as emerging views of the binding relationships between adaptor, APC core, and substrate.

\section{The TPR arm of the APC}

The APC has several copies of each member of a set of TPR-containing proteins thought to be important for association of the adaptor proteins with the APC. Three of

Table 1. Subunits of the APC

\begin{tabular}{lcccl}
\hline & & & APC subunit & \\
\hline S. cerevisiae & Human & S. pombe & Essential? & Known function/motifs \\
\hline Apc1 & Apc1 & Cut4 & Yes & Homology with Rpn1/2 \\
Apc4 & Apc4 & Lid1 & Yes & Required for TPR subunits to bind Apc1 \\
Apc5 & Apc5 & Apc5 & Yes & Required for TPR subunits to bind Apc1 \\
Apc2 & & & \\
Apc11 & Apc2 & Apc2 & Yes & Cullin domain, catalytic core, E2 binding \\
Doc1 & Apc11 & Apc11 & Yes & RING finger, catalytic core, E2 binding \\
Cdc23 & Apc10 & Apc10 & No & Doc domain, IR motif, promotes substrate binding \\
Cdc16 & & & & \\
Cdc27 & Apc8 & Cut23 & Yes & TPR, required for Cdc16 and Cdc27 to bind \\
- & Apc6 & Cut9 & Yes & TPR, required for Cdc27 to bind \\
Cdc26 & Apc3 & Nuc2 & Yes & TPR, interacts with Cdh1/Cdc20 adaptors \\
Apc9 & Apc7 & - & ND & TPR \\
Swm1 & & & \\
Mnd2 & Cdc26 & Hcn1 & No & Induced upon heat shock \\
- & - & - & No & Promotes TPR association \\
& Apc13 & Apc13 & No & Promotes TPR association \\
\hline Cdc20 & - & Apc15 & No & Regulation of Amal in meiosis \\
Cdh1 & - & Apc14 & No & \\
Ama1 & & & Adaptor subunit & \\
\hline Doc1 & Cdc20 & Slp1 & Yes & WD40 domain; substrate recognition \\
& Cdh1 & Ste9 & No & WD40 domain; substrate recognition
\end{tabular}

${ }^{a}$ Doc1 and Apc13 are essential in S. pombe but not S. cerevisiae. 
A

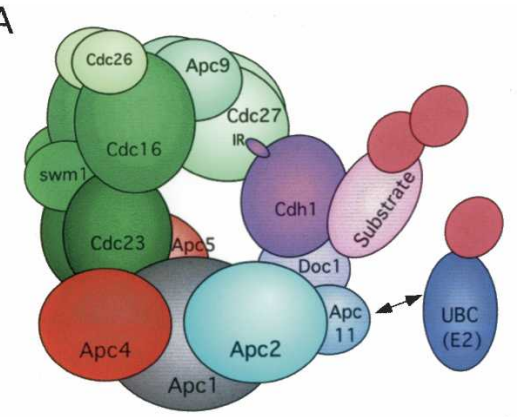

D

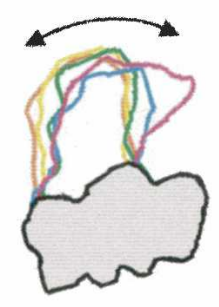

B

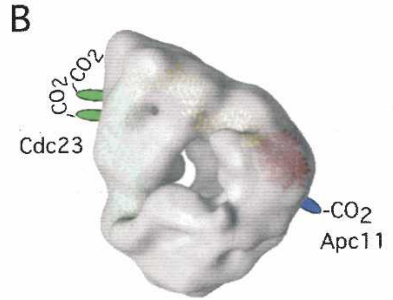

C Apc2

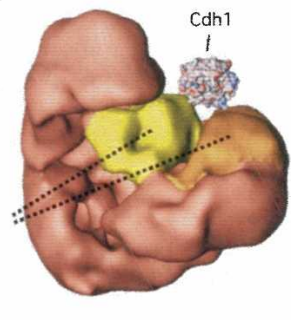

$E$

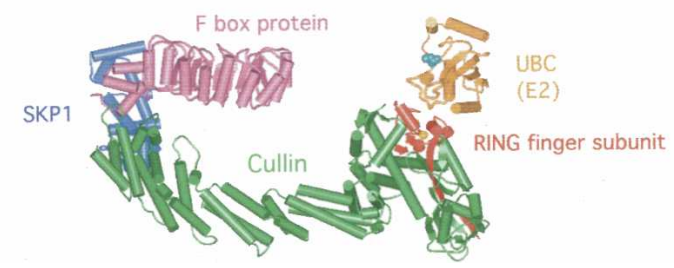

Figure 1. Structures of ubiquitin ligases. (A) An architectural map based on the known binding dependencies. The TPR arm is shown with two copies of each subunit, which have been observed in multiple systems. Each of the APC subunits, Cdh1 (purple), and ubiquitin (red), are shown to scale for size, whereas the E2 and substrate (pink) are shown as arbitrary sizes. Adapted from Thornton et al. (2006). (B) The EM structure of the budding yeast APC. The C-terminal tails of two copies of Cdc23 and one copy of Apc11 are placed on the structure based on immuno-EM analysis. Three TPR folds are modeled within the structure and are shown in green, yellow, and red. Reprinted from Passmore et al. (2005) with permission from Elsevier (C 2005). (C) The EM structure of the human APC, with Apc2 located based on immuno-gold staining. $(D)$ Outline of the human APC shown in the same orientation as in $C$. Colored outlines represent multiple angles observed for the "arc lamp" portion of the structure. $C$ and $D$ were reprinted from Dube et al. (2005) with permission from Elsevier (C 2005). (E) Crystal structure of the human SCF complex with the F-box protein Skp2. The active site cysteine of the E2 is shown in cyan in space-filling format. The E2, shown in gold ( $\mathrm{UbcH} 7)$, is docked onto the RING subunit (Rbx1, in red) based on the RING-E2 positioning in the c-Cbl-UbcH7 structure. Adapted by permission from Macmillan Publishers Ltd.: Nature (Zheng et al. 2002) (๔ 2002).

these, Cdc16, Cdc23, and Cdc27, are conserved from yeast to humans. A fourth TPR-containing subunit, Apc7, is the only subunit of the vertebrate APC not conserved to yeast. Several studies have suggested that the APC contains two to three copies of each of these subunits, although the exact stoichiometry is still not known (Lamb et al. 1994; Dube et al. 2005; Passmore et al. 2005). Moreover, these subunits are able to form a complex, together with Cdc26, independent of most of the remainder of the APC (Thornton et al. 2006). Cdc26 is a nonessential, small, heat-inducible APC subunit important for the APC's ability to maintain full association of Cdc27 and Cdc16 when cells are grown at high temperatures (Zachariae et al. 1996, 1998b). In addition to Cdc26, two other small, nonessential APC subunits, Swm 1 and Apc9, are also important for the integrity of the TPR complex at high temperatures (Zachariae et al. 1998b; Schwickart et al. 2004), although it is not yet known whether these are retained in the independent TPR subcomplex.

The three TPR subunits are tethered to Apc1, a large scaffolding subunit, through two subunits, Apc4 and Apc5 (Thornton et al. 2006). Apc4 and Apc5 appear to hold onto the TPR complex through Cdc23, and they do so in such a way that each of the three proteins' association with Apcl depends on the other two (Thornton et al. 2006). The TPR subunits bind in an ordered complex, such that $\mathrm{Cdc} 23$ associates with Cdc16, which in turn binds Cdc27 (Schwickart et al. 2004; Kraft et al. 2005; Thornton et al. 2006). The TPR complex may represent a subcomplex in the APC that is more evolutionarily dynamic. Given that the APC contains multiple copies of multiple TPR subunits, it is likely that these arose through duplication of the same ancestral protein. This phenomenon is highlighted by the fact that vertebrate APC has an additional TPR subunit, Apc7, and plants have a specialized, developmentally regulated version of Cdc27, called HOBBIT (Blilou et al. 2002).

The adaptor proteins Cdc20 (also called Fizzy), Cdh1 (also called Hct1 or Fizzy-related), and Amal contain two known domains that contribute to binding to the APC: an internal motif known as the "C box" (Schwab et al. 2001), and a C-terminal isoleucine and arginine (IR) (Vodermaier et al. 2003). This C-terminal motif, referred to as the IR motif for obvious reasons, extends beyond these two terminal residues to include a more loosely conserved hydrophobic region that extends an additional six amino acids. The IR motif has been conserved in these three adaptors in almost every species examined and promotes the association of the adaptor with the TPR subunits (Passmore et al. 2003; Vodermaier et al. 2003). TPR motifs associate with proteins through their C-terminal peptides in other contexts as well, such as the well characterized interaction between the TPR repeats of PEX5 and the C-terminal five amino acids of PTS1 (Gatto et al. 2000). Interestingly, one core APC subunit, Doc1, also contains a C-terminal IR, suggesting that it may make internal contacts with the TPR subunits. Doc1's IR has been shown to associate with Cdc27 in vitro (Wendt et al. 2001), although this interaction is clearly not the primary means with which either subunit is held in the APC complex (Thornton et al. 2006). Whatever the function of Doc1's IR domain is, it is clearly not required for Doc1's established role in promoting the processivity of the APC, as deletion of the $\mathrm{C}$ terminus of Doc1 has only a modest effect on this activity (Carroll et al. 2005).

In vitro binding studies have shown that the IR do- 
main of Cdh1 is required for wild-type levels of association with the APC (Passmore et al. 2003; Vodermaier et al. 2003). The IR tail of Cdh1 binds to both Cdc27 and the mammalian-specific TPR protein Apc7 in vitro (Vodermaier et al. 2003). However, Cdh1 clearly retains function in the absence of this IR/Cdc27 interaction in vivo and in vitro. The Cdh1- $\Delta$ IR protein is able to activate core APC in vitro almost as well as wild-type Cdh1 (Thornton et al. 2006), despite the fact that the CDH1$\Delta I R$ allele does not support growth in a mutant background that requires $C D H 1$ for viability (Kraft et al. 2005). Moreover, APC lacking Cdc27 (APC ${ }^{\mathrm{cdc} 27 \Delta}$ ) retains some activity in vivo and in vitro (Thornton and Toczyski 2003; Thornton et al. 2006). Taken together, these data suggest that the IR Cdc 27 interaction is important, but not essential for APC ${ }^{\mathrm{Cdh} 1}$ activity. Cdc20, Cdh1, and Amal homologs also contain a C-box domain important for interaction with the APC. The residual APC activity seen in $\mathrm{APC}^{\mathrm{cdc} 27 \Delta}$ is independent of the Cdh1's IR but appears to require its $\mathrm{C}$ box, since weak mutations in Cdh1's C box eliminate APC ${ }^{\text {cdc27 }}$ activity (Thornton et al. 2006).

Surprisingly, the IR domain is dispensable for functional interactions between the APC and Cdc20: CDC20-IRA strains grow at approximately wild-type rates and turn over CDC20-dependent substrates (Thornton et al. 2006). However, the IR domain appears to be important for APC-mediated turnover of Cdc20 itself, and this again seems to be mediated through the TPR subunit Cdc27 and is independent of Cdh1 (Thornton et al. 2006). This is consistent with earlier data indicating that Cdc20 is also targeted by the APC through a second "destruction-box (D-box)-independent" mode (Prinz et al. 1998). This direct E3-mediated turnover of the APC adaptor Cdc20 may be analogous to the rapid turnover of F-box proteins by the SCF /Galan and Peter 1999).

The observation that there are multiple copies of each TPR protein within the APC opens up the possibility that multiple IR proteins could simultaneously be recruited through Cdc27, or any of the other TPR proteins, within a single APC complex. This could serve simply to increase the efficiency through which substrates can interact with these adaptors. In some instances, this second IR docking site could serve to promote the targeting of a substrate to the APC. Recently, it has been reported that one substrate, Nek2A, associates with the APC in vitro through a C-terminal tail similar to the IR domain (Hayes et al. 2006). Yet, the degradation of this substrate is dependent on Cdc20, suggesting that both this substrate and Cdc 20 might associate with TPR subunits simultaneously, either through two copies of the same TPR subunit or through different TPR subunits. The mechanism of Nek2A targeting could also be related to the IR-dependent turnover of Cdc20 by the APC. Future experiments will determine whether this turnover is mediated in trans by a neighboring Cdc20 on the same complex or directly by the APC, such that Cdc20 would play only a passive role in this process as a substrate.

\section{The Cullin RING core: the belly of the beast}

The APC is the most structurally complex member of this Cullin-RING family of multisubunit ubiquitin ligases, as it contains one or more copies of 13 individual subunits (Table 1). However, the APC is likely to share mechanistic features of the simpler members of this class. Indeed, the APC's cullin and RING domain proteins (Apc2 and Apc11, respectively) have been shown to be sufficient for a modest level of enzymatic activity in vitro, albeit at the expense of specificity, suggesting that the large number of other subunits are involved in various forms of regulation (Gmachl et al. 2000; Leverson et al. 2000; Tang et al. 2001). The best-understood member of the Cullin-RING family is the SCF complex, a ubiquitin ligase that also plays an important role in cell division by targeting CDK inhibitors and some G1 cyclins, among other proteins, for degradation (for review, see Willems et al. 2004).

The SCF holoenzyme has three core subunits: a RING finger protein, a cullin, and Skp1, a linker that attaches the cullin to one of many F-box proteins. F-box proteins serve as substrate-binding subunits and associate with cullin through a conserved domain called an $\mathrm{F}$ box (Skowyra et al. 1997). Interactions between the F-box subunit and the substrate are often mediated by substrate modification (typically phosphorylation) (Willems et al. 2004). The crystal structure of the SCF has provided insight into the mechanism of the enzyme (Fig. 1E; Zheng et al. 2002). This structure revealed that the cullin subunit forms a rigid rod, with the RING subunit and E2 bound to one end and the Skp1 and F-box adaptor protein bound to the other (Zheng et al. 2002). The cullin repeats form the central rod, while the C-terminal winged helix motif associates with the RING subunit. Despite the fact that the cullin subunits of the SCF and APC do not have sequence conservation outside the cullin homology domain, a crystal structure of the $\mathrm{C}$ terminus of APC2 showed that it formed a Winged helix domain superimposable on that of the SCF's cullin (Zheng et al. 2002). The SCF's extended structure places the F-box adaptor protein $\sim 50 \AA$ away from the E2. Since the F box is known to bind directly to substrate, a reasonable model is that the substrate fits into this 50 - $\AA$ gap, creating a "hot zone" where the transfer of ubiquitin from E2 to substrate is promoted.

The function of the RING finger itself in this process is, in part, recruitment of the E2. The cocrystal structure of $\mathrm{Cbl}$ (a monomeric RING finger E3) and its E2 show that the RING finger directly associates with the E2 (Zheng et al. 2000). Moreover, the residues in Cbl's RING that interact with the E2 are conserved in both the SCF and APC RING subunits (Zheng et al. 2002). In addition to merely recruiting E2s, the SCF RING finger subunit has been suggested to activate E2. E2s are capable of polyubiquitinating themselves in the absence of substrate. This reaction is promoted by incubation of the E2 with its E3 in a way that requires RING finger proteins, opening the possibility that the E2 is in some way activated (Seol et al. 1999; Skowyra et al. 1999). The 
autoubiquitination activity of E2s may be important biologically in the case of the APC. Unlike the SCF, which is thought to use a single E2, the APC is able to use several E2s (for review, see Peters 2002). Interestingly, E2s have been suggested to function with a substrate preference in vertebrates; UbcH10 appears to function more efficiently on cyclin A than does UbcH5 (Fig. 2; Rape and Kirschner 2004). These investigators further propose that the autoubiquitination of $\mathrm{UbcH} 10$ is initially blocked when it is engaged in $\mathrm{APC}^{\mathrm{Cdh} 1}$-mediated destruction of substrates in mitosis. When the APC has lowered the levels of these substrates significantly, in G1, UbcH10 autoubiquitination is then increased, leading to its turnover. This results in accumulation of cyclin A, which in turn further inactivates the $\mathrm{APC}^{\mathrm{Cdh} 1}$.

Autoubiquitination of E2s may also provide insights into the mechanism of E3s. Kinetic analyses have suggested that ubiquitin-charged E2s associate with the SCF quite loosely, allowing them to travel across the $50-\AA$ gap to reach their substrate (Deffenbaugh et al. 2003). This would allow the SCF to target substrates of a range of sizes despite their distance from the RING-bound E2. It should be noted that the cullin-based linker separating the RING from the substrate is unlikely to facilitate this interaction by arching, given the rigidity inferred from its structure. In fact, placement of a flexible linker in this cullin domain has been shown to interfere with SCF function (Zheng et al. 2002). The APC is also thought to cycle charged E2s during its ubiquitination of a single substrate, as opposed to recharging the same E2 throughout the reaction (Carroll and Morgan 2002). Unlike the rigidity observed in the SCF, the EM structure of the vertebrate APC shows multiple forms in which the upper "lamphead" portion of the structure appears to move up and down on the body of the complex (Fig. 1D; Dube et al. 2005). This flexibility might facilitate the APC's association with its great variety of substrates.

Not only does the APC act on a large number of substrates of different sizes, it also acts on those substrates to elongate the ubiquitin chain. This may require some alteration of the positioning of the substrate by the APC, which could be mediated by conformational flexibility of the complex. Polyubiquitination adds another layer of specificity to the reaction: The APC must both be specific for its target substrate and extend that target's ubiquitin chain; in addition, polyubiquitin chains can be extended from several different lysines on the ubiquitin (for review, see Hochstrasser 2006). While the APC has conventionally been thought to create Lys 48 linkage (K48) exclusively, some recent data have suggested that the APC generates many non-K48-linked ubiquitin chains in vitro, and that these products can also target substrate to the proteasome (Kirkpatrick et al. 2006). E2s are likely to provide specificity for the ubiquitin linkage; for example, it has been shown that domains on E2s themselves are important for linkage specificity (Petroski and Deshaies 2005). Moreover, several E2s have been shown to generate unanchored ubiquitin chains of a specific linkage (e.g., K48 vs. K63 linkages) in the absence of an E3 (Chen and Pickart 1990; Van Nocker and Vierstra 1991; Hofmann and Pickart 1999).

Recent cryo-EM structures of human, Xenopus, and Saccharomyces cerevisiae APC reveal that the enzyme has an overall triangular shape with a large inner cavity (Dube et al. 2005; Passmore et al. 2005). This cavity appears to be too small to accommodate an E2, substrate, and ubiquitin, and therefore is unlikely to represent a "reaction chamber" in which the ubiquitination occurs. The cavity does appear to be present in APC from all three organisms studied, suggesting that it serves some as yet undetermined role in the enzyme's function. This

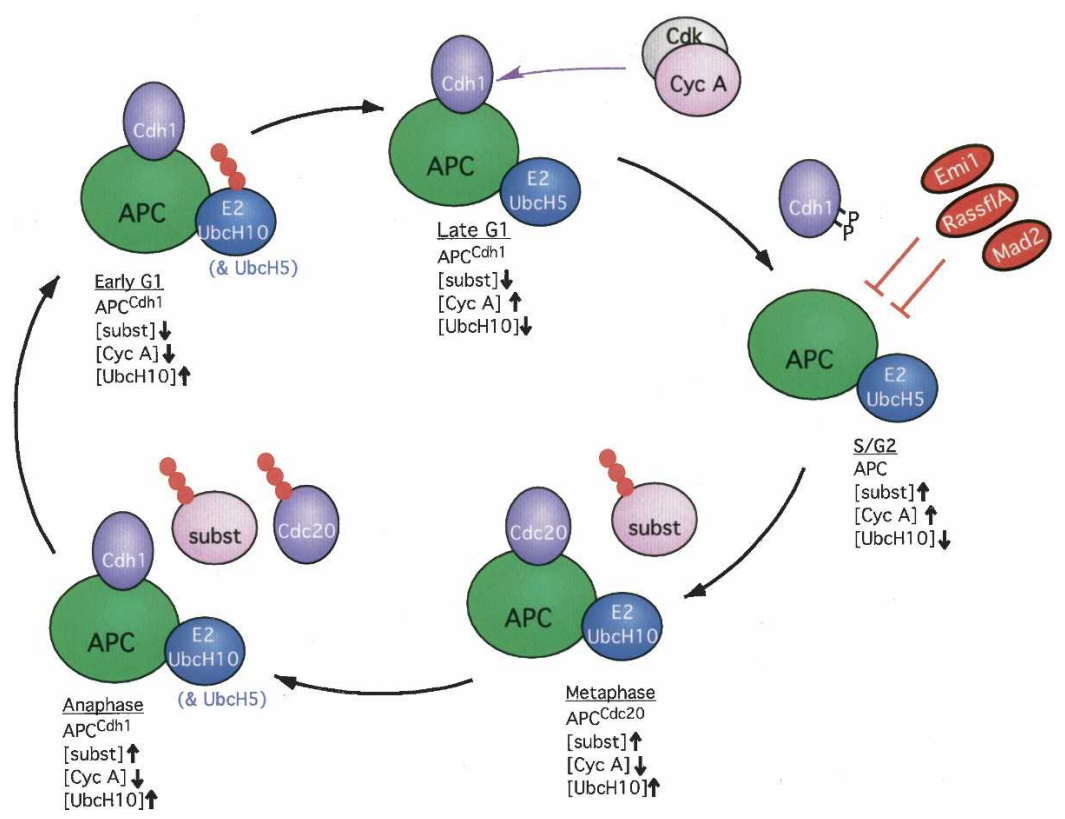

Figure 2. A model depicting the regulation of APC activity. In early G1, most mitotic substrates (subst) have been degraded. This allows $\mathrm{APC}^{\mathrm{Cdh} 1}$ to promote $\mathrm{UbcH} 10$ polyubiquitination, leading to its destruction. UbcH5 is stable and remains APC-associated, but does not target Cyclin A (Cyc A) efficiently. Thus, by late G1, Cyclin A levels can increase and promote inactivation of Cdh1. The APC remains inactive in S and G2 largely through inhibition by several APC inhibitors (in red). By metaphase, these inactivators have themselves become inactivated, allowing accumulation of Cdc20 and UbcH10. This drives down cyclin A, which helps promote the degradation of $\mathrm{Cdc} 20$ and the reactivation of Cdh1 during anaphase. 
means that the enzymatic reaction itself must occur somewhere on the surface of the enzyme. However, where this site is remains to be determined, as there is no clear indication from the EM structures of a cullin scaffold like the one seen in the SCF.

Taken together, the core APC is a large enzyme that can be divided into two subcomplexes bound to an Apc1/ Apc4/Apc5 scaffold (Fig. 1A). The first is the Apc2/ Apc11/Doc1 complex, which binds directly to Apc1 and recruits the E2. The second is the TPR subcomplex, and consists of the TPR proteins and the nonessential subunits Swm1, Cdc26, and Apc9. The TPR complex clearly has an important role in recruiting the adaptors Cdh1 and Cdc20, and thus bringing in the substrate /discussed below). The TPR complex binds to Apc1 through Apc4 and Apc5, independently of the cullin/RING Apc2/ Apc11/Doc1 arm. Together, these two arms are able to position the E2 and the substrate in such a way as to promote ubiquitin transfer.

\section{Substrate binding: the adaptors' big dance}

Both the APC and the SCF regulate cell cycle progression. These ligases differ fundamentally in that SCF activity is thought to be regulated largely at the substrate level, whereas the APC is regulated in the activity of the E3 enzyme itself. The SCF is thought to be active throughout the cell cycle, and, for those substrates examined in detail, ubiquitinates targets only after they are modified. For example, the Cdk inhibitor Sicl appears in late mitosis, but is only targeted for degradation by the SCF when it is multiply phosphorylated at the G1/S transition by CDK associated with the yeast G1 cyclins (Nash et al. 2001). In contrast, the periodic targeting of substrates by the APC is mediated, largely, through its regulated association with the adaptor proteins. However, there are exceptions to this. Ubiquitination of three APC substrates (yeast securin, and vertebrate Cdc6 and Aurora kinase) is inhibited by their phosphorylation (Littlepage and Ruderman 2002; Agarwal et al. 2003; Mailand and Diffley 2005).

Cdh1's association with the APC is negatively controlled by phosphorylation of Cdh1 by Cdk. Yeast $A P C^{C d h 1}$ is active from late mitosis until G1/S, when cyclin B/Cdk activity is low and thus Cdh1 is unphosphorylated (Zachariae et al. 1998a; Jaspersen et al. 1999). Similarly, inhibition of vertebrate Cdh1 by phosphorylation is mediated by cyclin A/Cdk2 (Fig. 2; Lukas et al. 1999; Kramer et al. 2000). Since Cdh1 is largely responsible for reducing $\mathrm{Cdk} /$ cyclin B activity, this mode of regulation constitutes a positive feedback loop. The regulation of Cdc20-dependent APC activity is more complicated. First, Cdc20 is itself a target of the APC, and is destabilized between mitosis and G1/S (Prinz et al. 1998; Shirayama et al. 1998). Second, it is thought that phosphorylation of the three TPR subunits of the APC by CDK promotes Cdc20 binding. In budding yeast, each of the three TRP subunits is phosphorylated by Cdk (Rudner and Murray 2000). In mammalian cells, Apc1 as well as the four TPR proteins have been shown to be Cdk targets (Kraft et al. 2003). While this has been suggested to aid in regulating the $\mathrm{APC} / \mathrm{Cdc} 20$ association, it is clearly not required in yeast: Simultaneous mutation of all of the Cdk sites on the APC (on Cdc16, Cdc27, and Cdc23) in yeast is not lethal, whereas deletion of the CDC20 gene is (Rudner and Murray 2000). In addition, Cdc20 has been reported to be negatively regulated by phosphorylation in response to DNA damage and spindle perturbation (Searle et al. 2004; Tang et al. 2004).

Finally, both $\mathrm{APC}^{\mathrm{Cdc} 20}$ and $\mathrm{APC}^{\mathrm{Cdh} 1}$ are directly inhibited by any of several APC inhibitors, many of which show some specificity for Cdc20 or Cdh1 complexes. The spindle assembly checkpoint inhibits $\mathrm{APC}^{\mathrm{Cdc} 20}$ in response to unattached kinetochores. Mad2 and BubR1 are able to directly inhibit $\mathrm{APC}{ }^{\mathrm{CDC} 20}$ in vitro (Sudakin et al. 2001; Fang 2002), and along with Bub3 exist in a complex with Cdc20. This complex, called the MCC, can be found in both yeast and metazoans (Hardwick et al. 2000; Sudakin et al. 2001). The exact mechanism by which the spindle checkpoint is activated is not known; however, data are accumulating supporting a very interesting model in which Mad2 can exist as either of two conformers, only one of which is active (Yu 2006). The spindle checkpoint also destabilizes Cdc20 in response to spindle insults (Pan and Chen 2004); this effect is not dependent on Cdh1 or the D box (see below) of Cdc20. The related Mad2B protein inhibits $\mathrm{APC}^{\mathrm{Cdh} 1}$ (Chen and Fang 2001; Pfleger et al. 2001b), but does not appear to be associated with the spindle checkpoint. Both Mad2 and $\mathrm{Mad} 2 \mathrm{~B}$ are unable to inhibit preformed $\mathrm{APC}^{\mathrm{Cdc} 20}$ and $\mathrm{APC}^{\mathrm{Cdh} 1}$ complexes. In contrast, the Emil protein can inhibit preformed $\mathrm{APC}^{\mathrm{Cdc} 20}$ and $\mathrm{APC}^{\mathrm{Cdh} 1}$ complexes, possibly by acting as an APC pseudosubstrate (Reimann et al. 2001; Miller et al. 2006). Another Emil-related $\mathrm{APC}^{\mathrm{Cdc} 20}$ inhibitor, XErp1, has recently been identified and appears to act in a parallel pathway to Emil (Schmidt et al. 2005). Several other APC inhibitors have also emerged in recent years. The tumor suppressor RASSF1A inhibits APC ${ }^{\mathrm{Cdc} 20}$ activity sometime in prometaphase, contributing to the accumulation of mitotic cyclins (Song et al. 2004). The Rae1/Nup98 complex blocks the ability of $\mathrm{APC}^{\mathrm{Cdh} 1}$ to ubiquitinate securin, but does not block substrate binding (Jeganathan et al. 2005, 2006), perhaps priming securin for rapid destruction by prebinding with inhibited complexes. These results demonstrate that inhibitors of the APC have evolved to block enzyme activity at various steps and with varying degrees of specificity; as we learn more about the mechanism of these inhibitors, it may teach us important lessons about how the APC-adaptor-substrate complex forms and functions.

In addition to Cdc 20 and $\mathrm{Cdh} 1$, several organisms have been found to encode a third, meiosis-specific adaptor, called Amal in budding yeast. Amal is required for spore formation, and $\mathrm{APC}^{\mathrm{Ama1}}$ also targets at least some $\mathrm{APC}^{\mathrm{Cdc} 20}$ substrates, such as securin (Oelschlaegel et al. 2005; Penkner et al. 2005). Interestingly, Ama1-mediated APC activity is negatively regulated by $\mathrm{Mnd} 2$, a subunit of the "core APC" (Oelschlaegel et al. 2005; Penkner et al. 2005). Mnd2 was recently discovered in budding and 
fission yeast as a core APC component in mitotic cells (Yoon et al. 2002; Hall et al. 2003). It is not essential for mitosis in either yeast, although cells lacking Mnd2 grow slowly in S. cerevisiae and are synthetically lethal with other APC mutants in Schizosaccharomyces pombe, suggesting that is does have a mitotic role in APC function (Yoon et al. 2002; Hall et al. 2003). Mnd2 is phosphorylated during meiosis and is degraded at anaphase II of meiosis, resulting in an increase in APC Ama1 activity (Oelschlaegel et al. 2005; Penkner et al. 2005).

Cdh1 and Cdc20 typically recognize substrates through motifs found within those substrates. The two best described of these are the D box (Glotzer et al. 1991) and the KEN box (Pfleger and Kirschner 2000), although other less well characterized motifs, such as the A box (Littlepage and Ruderman 2002) and the GxEN motif (Castro et al. 2003) have been described. The D box is a loosely defined sequence, R-X-X-L-X-X-X-X-N, while the $\mathrm{KEN}$ box is defined as K-E-N-X-X-X-E/D/N. APC substrates can be targeted by a KEN box, a D box, both, or even multiple $\mathrm{D}$ boxes. In some cases, the targeting domains for a substrate have even been swapped during evolution; Cdc20 contains two D boxes in yeast, but a KEN box in mammalian cells. These substrate-targeting motifs appear to be able to function for both adaptors. While the KEN box was originally identified as a targeting motif for $\mathrm{APC}^{\mathrm{Cdh} 1}$, it has been shown to be recognized by $\mathrm{APC}^{\mathrm{Cdc} 20}$ in some cases (Burton and Solomon 2001) but not others (Passmore et al. 2003). Recently, substrates have also been shown to be targeted in vitro to the APC by an interaction between the core APC and a C-terminal IR motif in the substrate (Hayes et al. 2006). Such interactions were previously known to be important for the association between the adaptors and the APC.

Early studies showed that substrates are recognized by the adaptors Cdc20/Cdh1, which serve largely as substrate recruiters by binding to substrates at a number of conserved motifs (Burton and Solomon 2001; Hilioti et al. 2001; Pfleger et al. 2001a; Schwab et al. 2001; Kraft et al. 2005). Several lines of evidence now suggest that the core APC itself also recognizes substrates. The core APC binds quantitatively to D-box affinity columns (Yamano et al. 2004). Binding of substrate to the APC could be mediated by the APC subunit Doc1, which has been shown to contribute to substrate recognition by the APC (Carroll and Morgan 2002; Passmore et al. 2003). Moreover, Doc1 appears to mediate its substrate association through the substrate's D box (Carroll et al. 2005). Although Doc1 is clearly important for substrate binding, it is not clear that Docl participates directly, since no direct interaction between Docl and substrate has been found despite extensive investigations. The ability of both the core APC and the adaptors to bind the D box suggests that they might associate with the D box at different stages of the ubiquitination reaction. The combination of substrate-binding motifs that a substrate possesses and its affinity to both the adaptors and/or the APC core may together dictate the processivity of the APC on substrates (Carroll and Morgan 2002). The difference in the processivity of APC on different sub- strates, in part, helps to dictate the order in which those substrates are targeted for degradation (Carroll and Morgan 2002; Rape et al. 2006).

Thus, data from several labs have shown that the core APC, the adaptors, and substrates are each able to directly interact with the other two, albeit weakly. Moreover, catalysis is clearly the result of these three coming together to form an $\mathrm{APC}^{\mathrm{Cdc} 20 / \mathrm{Cdh} 1}$-substrate complex. Kinetic data have shown that multiple E2s shuttle into this complex during the course of a polyubiquitination reaction, suggesting that E2 association is unlikely to be important for formation of the initial activated $\mathrm{APC}^{\mathrm{Cdc} 20 / \mathrm{Cdh} 1}$-substrate complex (Carroll and Morgan 2002). This leaves open the mechanistic question as to whether one of these three possible two-component subcomplexes forms first and recruits the third member. For example, does the core APC recognize a preassembled adaptor-substrate complex, or does the APC Cdc20/Cdh1 recognize free substrate? Mutagenesis of yeast Cdh1 identified both mutants that bind substrate and not the core APC, and mutants that bound neither substrate nor the core APC. However, it did not identify mutants that bound the core APC but did not bind substrate (Burton et al. 2005). These data are consistent with substrate binding promoting Cdh1's association with the APC, such that Cdh1 alleles that could not bind substrate would also necessarily be unable to bind the core APC. This would suggest that the Cdh1-substrate interaction would precede formation of the APC ${ }^{\mathrm{Cdc} 20 / \mathrm{Cdh}_{1}}$-substrate complex in vivo (Burton et al. 2005). While overexpression of a substrate (Hsll) fragment increased the association of Cdh1 with the APC in vivo (Burton et al. 2005), it did not significantly affect this association in vitro (Thornton et al. 2006). Alternatively, it has been suggested that adaptor may first bind APC and that this complex could then bind substrate. Gel shift assays have identified an APC-Cdh1 complex that is competent to bind substrate (Passmore and Barford 2005). Recent "isotope-trapping" experiments showed that human APC Cdc20 mixtures could trap substrate (make them resistant to chasing), whereas APC or Cdc20 alone could not, suggesting that the $\mathrm{APC}^{\mathrm{Cdc} 20}$ complex could interact with substrate in such a way as to form product in the face of excess cold substrate (Eytan et al. 2006). It is important to note, however, that each of these studies examines binding and/or in vitro activity. Further experiments will need to be performed to determine which is the pertinent subcomplex in vivo.

\section{Perspectives: Can the APC get any bigger?}

Recent work has shed considerable light on the structure and function of the APC. We now have a greater understanding of the stoichiometry and arrangement of the subunits, as well as critical clues about how the enzyme core, adaptors, substrate, and E2 may coordinate to form a functional enzyme. We understand that the APC has a modular structure that partially divides substrate binding and catalysis to different domains, and that the complex may take on two or more different conformations 
during catalysis. The adaptor-substrate complex that was once thought to be a simple shepherding relationship clearly involves subtle interaction codependencies that will require further study, both genetic and biochemical, to fully understand. Above and beyond this puzzle, however, is the possibility that the APC is organized into a higher-order structure, and that this structure plays a key role in the enzyme's mechanism.

Passmore and Barford (2005) observed that purified APC migrated as a doublet on nondenaturing gels. The larger, slower-migrating form was roughly twice as large as most previous estimates of the size of the APC. The dimeric and monomeric forms appear quite stable, since they can be separated on glycerol gradients and subsequently run on native gels to yield homogenously electrophoresing monomer and dimer complexes. In addition, careful perusal of purified APC by TEM and negative staining revealed many cases where two complexes appeared to be in direct contact or connected by a thin linker. Both forms were able to bind substrate and ubiquitinate it, but the apparently dimeric form of the APC was substantially more active toward Pds1 or Hsl1, largely due to an increase in the processivity of the enzyme (Passmore et al. 2005).

How might an APC dimer assemble? One possibility is that the Docl subunit serves as the link between two complexes. Prior reports suggested that Doc1 is capable of binding Cdc27 (Wendt et al. 2001), yet Doc1 binding to the APC is dependent on Apc2 and not on Cde27 (Thornton et al. 2006). Thus, Doc1 may make contacts with APC subunits outside those that lead to its association with the complex. This could mediate an interaction between two APC complexes, where Doc1 from one complex interacts weakly with a subunit from the other complex. This model is particularly compelling when one considers the difference in activity of dimers and monomers in light of the established role of Doc1 in APC processivity (Carroll and Morgan 2005). Alternatively, the observation that multiple copies of each TPR subunit are present within the APC could be hinting that these subunits self-associate. The availability of mutant complexes that lack such domains or subunits will likely prove valuable in elucidating such higher-order structures, and how they impact on the function of the APC.

\section{Acknowledgments}

We thank J. Benanti, C. Bonilla, M. Matyskiela, D. Morgan, and M. Rodrigo-Brenni for critical reading of the manuscript; D. Barford, L. Passmore, N. Pavletich, and H. Stark for the generous use of images; and M. Rape for useful discussions. This work was supported by a grant from the National Institutes of Health (GM070539).

\section{References}

Agarwal, R., Tang, Z., Yu, H., and Cohen-Fix, O. 2003. Two distinct pathways for inhibiting pds1 ubiquitination in response to DNA damage. J. Biol. Chem. 278: 45027-45033.

Blilou, I., Frugier, F., Folmer, S., Serralbo, O., Willemsen, V., Wolkenfelt, H., Eloy, N.B., Ferreira, P.C., Weisbeek, P., and Scheres, B. 2002. The Arabidopsis HOBBIT gene encodes a CDC27 homolog that links the plant cell cycle to progres- sion of cell differentiation. Genes \& Dev. 16: 2566-2575.

Burton, J.L. and Solomon, M.J. 2001. D box and KEN box motifs in budding yeast Hsllp are required for APC-mediated degradation and direct binding to Cdc20p and Cdh1p. Genes \& Dev. 15: 2381-2395.

Burton, J.L., Tsakraklides, V., and Solomon, M.J. 2005. Assembly of an APC-Cdh1-substrate complex is stimulated by engagement of a destruction box. Mol. Cell 18: 533-542.

Carroll, C.W. and Morgan, D.O. 2002. The Doc1 subunit is a processivity factor for the anaphase-promoting complex. Nat. Cell Biol. 4: 880-887.

Carroll, C.W. and Morgan, D.O. 2005. Enzymology of the anaphase-promoting complex. Methods Enzymol. 398: 219-230.

Carroll, C.W., Enquist-Newman, M., and Morgan, D.O. 2005. The APC subunit Doc1 promotes recognition of the substrate destruction box. Curr. Biol. 15: 11-18.

Castro, A., Vigneron, S., Bernis, C., Labbe, J.C., and Lorca, T. 2003. Xkid is degraded in a D-box, KEN-box, and A-boxindependent pathway. Mol. Cell. Biol. 23: 4126-4138.

Chen, J. and Fang, G. 2001. MAD2B is an inhibitor of the anaphase-promoting complex. Genes \& Dev. 15: 1765-1770.

Chen, Z. and Pickart, C.M. 1990. A 25-kilodalton ubiquitin carrier protein (E2) catalyzes multi-ubiquitin chain synthesis via lysine 48 of ubiquitin. J. Biol. Chem. 265: 21835-21842.

Cohen-Fix, O., Peters, J.M., Kirschner, M.W., and Koshland, D. 1996. Anaphase initiation in Saccharomyces cerevisiae is controlled by the APC-dependent degradation of the anaphase inhibitor Pds1p. Genes \& Dev. 10: 3081-3093.

Deffenbaugh, A.E., Scaglione, K.M., Zhang, L., Moore, J.M., Buranda, T., Sklar, L.A., and Skowyra, D. 2003. Release of ubiquitin-charged Cdc34-S-Ub from the RING domain is essential for ubiquitination of the SCF(Cdc4)-bound substrate Sic1. Cell 114: 611-622.

Dube, P., Herzog, F., Gieffers, C., Sander, B., Riedel, D., Muller, S.A., Engel, A., Peters, J.M., and Stark, H. 2005. Localization of the coactivator Cdh1 and the cullin subunit Apc2 in a cryo-electron microscopy model of vertebrate APC/C. Mol. Cell 20: 867-879.

Eytan, E., Moshe, Y., Braunstein, I., and Hershko, A. 2006. Roles of the anaphase-promoting complex/cyclosome and of its activator Cdc20 in functional substrate binding. Proc. Nat1. Acad. Sci. 103: 2081-2086.

Fang, G. 2002. Checkpoint protein BubR1 acts synergistically with Mad2 to inhibit anaphase-promoting complex. Mol. Biol. Cell 13: 755-766.

Galan, J.M. and Peter, M. 1999. Ubiquitin-dependent degradation of multiple F-box proteins by an autocatalytic mechanism. Proc. Nat1. Acad. Sci. 96: 9124-9129.

Gatto Jr., G.J., Geisbrecht, B.V., Gould, S.J., and Berg, J.M. 2000. Peroxisomal targeting signal-1 recognition by the TPR domains of human PEX5. Nat. Struct. Biol. 7: 1091-1095.

Gieffers, C., Dube, P., Harris, J.R., Stark, H., and Peters, J.M. 2001. Three-dimensional structure of the anaphase-promoting complex. Mol. Cell 7: 907-913.

Glotzer, M., Murray, A.W., and Kirschner, M.W. 1991. Cyclin is degraded by the ubiquitin pathway. Nature 349: 132-138.

Gmachl, M., Gieffers, C., Podtelejnikov, A.V., Mann, M., and Peters, J.M. 2000. The RING-H2 finger protein APC11 and the E2 enzyme UBC4 are sufficient to ubiquitinate substrates of the anaphase-promoting complex. Proc. Nat1. Acad. Sci. 97: 8973-8978.

Hall, M.C., Torres, M.P., Schroeder, G.K., and Borchers, C.H. 2003. Mnd2 and Swm1 are core subunits of the Saccharomyces cerevisiae anaphase-promoting complex. I. Biol. Chem. 278: 16698-16705.

Hardwick, K.G., Johnston, R.C., Smith, D.L., and Murray, A.W. 
2000. MAD3 encodes a novel component of the spindle checkpoint which interacts with Bub3p, Cdc20p, and Mad2p. J. Cell Biol. 148: 871-882.

Hayes, M.J., Kimata, Y., Wattam, S.L., Lindon, C., Mao, G., Yamano, H., and Fry, A.M. 2006. Early mitotic degradation of Nek2A depends on Cdc20-independent interaction with the APC/C. Nat. Cell Biol. 8: 607-614.

Herzog, F. and Peters, J.M. 2005. Large-scale purification of the vertebrate anaphase-promoting complex/cyclosome. Methods Enzymol. 398: 175-195.

Hilioti, Z., Chung, Y.S., Mochizuki, Y., Hardy, C.F., and CohenFix, O. 2001. The anaphase inhibitor Pds1 binds to the APC/ $\mathrm{C}$-associated protein Cdc20 in a destruction box-dependent manner. Curr. Biol. 11: 1347-1352.

Hochstrasser, M. 2006. Lingering mysteries of ubiquitin-chain assembly. Cell 124: 27-34.

Hofmann, R.M. and Pickart, C.M. 1999. Noncanonical MMS2encoded ubiquitin-conjugating enzyme functions in assembly of novel polyubiquitin chains for DNA repair. Cell 96: 645-653.

Irniger, S., Piatti, S., Michaelis, C., and Nasmyth, K. 1995. Genes involved in sister chromatid separation are needed for B-type cyclin proteolysis in budding yeast. Cell 81: 269-278.

Jaspersen, S.L., Charles, J.F., and Morgan, D.O. 1999. Inhibitory phosphorylation of the APC regulator Hctl is controlled by the kinase Cdc28 and the phosphatase Cdc14. Curr. Biol. 9: 227-236.

Jeganathan, K.B., Malureanu, L., and van Deursen, J.M. 2005. The Rae1-Nup98 complex prevents aneuploidy by inhibiting securin degradation. Nature 438: 1036-1039.

Jeganathan, K.B., Baker, D.J., and van Deursen, J.M. 2006. Securin associates with APCCdh1 in prometaphase but its destruction is delayed by Rael and Nup98 until the metaphase/anaphase transition. Cell Cycle 5: 366-370.

King, R.W., Peters, J.M., Tugendreich, S., Rolfe, M., Hieter, P., and Kirschner, M.W. 1995. A 20S complex containing CDC27 and CDC16 catalyzes the mitosis-specific conjugation of ubiquitin to cyclin B. Cell 81: 279-288.

Kirkpatrick, D.S., Hathaway, N.A., Hanna, J., Elsasser, S., Rush, J., Finley, D., King, R.W., and Gygi, S.P. 2006. Quantitative analysis of in vitro ubiquitinated cyclin $\mathrm{B} 1$ reveals complex chain topology. Nat. Cell Biol. 8: 700-710.

Kraft, C., Herzog, F., Gieffers, C., Mechtler, K., Hagting, A., Pines, J., and Peters, J.M. 2003. Mitotic regulation of the human anaphase-promoting complex by phosphorylation. EMBO J. 22: 6598-6609.

Kraft, C., Vodermaier, H.C., Maurer-Stroh, S., Eisenhaber, F., and Peters, J.M. 2005. The WD40 propeller domain of Cdh1 functions as a destruction box receptor for APC/C substrates. Mol. Cell 18: 543-553.

Kramer, E.R., Scheuringer, N., Podtelejnikov, A.V., Mann, M., and Peters, J.M. 2000. Mitotic regulation of the APC activator proteins CDC20 and CDH1. Mol. Biol. Cell 11: 15551569.

Lamb, J.R., Michaud, W.A., Sikorski, R.S., and Hieter, P.A. 1994. Cdc16p, Cdc23p and Cdc27p form a complex essential for mitosis. EMBO J. 13: 4321-4328.

Leverson, J.D., Joazeiro, C.A., Page, A.M., Huang, H., Hieter, P., and Hunter, T. 2000. The APC11 RING-H2 finger mediates E2-dependent ubiquitination. Mol. Biol. Cell 11: 2315-2325.

Littlepage, L.E. and Ruderman, J.V. 2002. Identification of a new $\mathrm{APC} / \mathrm{C}$ recognition domain, the $\mathrm{A}$ box, which is required for the Cdh1-dependent destruction of the kinase Aurora-A during mitotic exit. Genes \& Dev. 16: 2274-2285.

Lukas, C., Sorensen, C.S., Kramer, E., Santoni-Rugiu, E., Lindeneg, C., Peters, J.M., Bartek, J., and Lukas, J. 1999. Accu- mulation of cyclin B1 requires E2F and cyclin-A-dependent rearrangement of the anaphase-promoting complex. Nature 401: 815-818.

Mailand, N. and Diffley, J.F. 2005. CDKs promote DNA replication origin licensing in human cells by protecting Cde6 from APC/C-dependent proteolysis. Cell 122: 915-926.

Miller, J.J., Summers, M.K., Hansen, D.V., Nachury, M.V., Lehman, N.L., Loktev, A., and Jackson, P.K. 2006. Emil stably binds and inhibits the anaphase-promoting complex/cyclosome as a pseudosubstrate inhibitor. Genes \& Dev. 20: 2410-2420.

Murray, A.W. 2004. Recycling the cell cycle: Cyclins revisited. Cell 116: 221-234.

Nash, P., Tang, X., Orlicky, S., Chen, Q., Gertler, F.B., Mendenhall, M.D., Sicheri, F., Pawson, T., and Tyers, M. 2001. Multisite phosphorylation of a CDK inhibitor sets a threshold for the onset of DNA replication. Nature 414: 514-521.

Oelschlaegel, T., Schwickart, M., Matos, J., Bogdanova, A., Camasses, A., Havlis, J., Shevchenko, A., and Zachariae, W. 2005. The yeast APC/C subunit Mnd2 prevents premature sister chromatid separation triggered by the meiosis-specific APC/C-Ama1. Cell 120: 773-788.

Pan, J. and Chen, R.H. 2004. Spindle checkpoint regulates Cdc20p stability in Saccharomyces cerevisiae. Genes \& Dev. 18: 1439-1451.

Passmore, L.A. and Barford, D. 2005. Coactivator functions in a stoichiometric complex with anaphase-promoting complex/ cyclosome to mediate substrate recognition. EMBO Rep. 6: 873-878.

Passmore, L.A., McCormack, E.A., Au, S.W., Paul, A., Willison, K.R., Harper, J.W., and Barford, D. 2003. Doc1 mediates the activity of the anaphase-promoting complex by contributing to substrate recognition. EMBO J. 22: 786-796.

Passmore, L.A., Booth, C.R., Venien-Bryan, C., Ludtke, S.J., Fioretto, C., Johnson, L.N., Chiu, W., and Barford, D. 2005. Structural analysis of the anaphase-promoting complex reveals multiple active sites and insights into polyubiquitylation. Mol. Cell 20: 855-866.

Penkner, A.M., Prinz, S., Ferscha, S., and Klein, F. 2005. Mnd2, an essential antagonist of the anaphase-promoting complex during meiotic prophase. Cell 120: 789-801.

Peters, J.M. 2002. The anaphase-promoting complex: Proteolysis in mitosis and beyond. Mol. Cell 9: 931-943.

Petroski, M.D. and Deshaies, R.J. 2005. In vitro reconstitution of SCF substrate ubiquitination with purified proteins. Methods Enzymol. 398: 143-158.

Pfleger, C.M. and Kirschner, M.W. 2000. The KEN box: An APC recognition signal distinct from the D box targeted by Cdh1. Genes \& Dev. 14: 655-665.

Pfleger, C.M., Lee, E., and Kirschner, M.W. 2001a. Substrate recognition by the Cdc20 and Cdh1 components of the anaphase-promoting complex. Genes \& Dev. 15: 2396-2407.

Pfleger, C.M., Salic, A., Lee, E., and Kirschner, M.W. 2001b. Inhibition of Cdh1-APC by the MAD2-related protein MAD2L2: A novel mechanism for regulating Cdh1. Genes \& Dev. 15: 1759-1764.

Pickart, C.M. 2001. Mechanisms underlying ubiquitination. Annu. Rev. Biochem. 70: 503-533.

Prinz, S., Hwang, E.S., Visintin, R., and Amon, A. 1998. The regulation of $\mathrm{Cdc} 20$ proteolysis reveals a role for APC components Cdc23 and Cdc27 during S phase and early mitosis. Curr. Biol. 8: 750-760.

Rape, M. and Kirschner, M.W. 2004. Autonomous regulation of the anaphase-promoting complex couples mitosis to S-phase entry. Nature 432: 588-595.

Rape, M., Reddy, S.K., and Kirschner, M.W. 2006. The proces- 
sivity of multiubiquitination by the APC determines the order of substrate degradation. Cell 124: 89-103.

Reed, S.I. 2003. Ratchets and clocks: The cell cycle, ubiquitylation and protein turnover. Nat. Rev. Mol. Cell Biol. 4: 855-864.

Reimann, J.D., Gardner, B.E., Margottin-Goguet, F., and Jackson, P.K. 2001. Emil regulates the anaphase-promoting complex by a different mechanism than Mad2 proteins. Genes \& Dev. 15: 3278-3285.

Rudner, A.D. and Murray, A.W. 2000. Phosphorylation by Cdc28 activates the Cdc20-dependent activity of the anaphase-promoting complex. J. Cell Biol. 149: 1377-1390.

Schmidt, A., Duncan, P.I., Rauh, N.R., Sauer, G., Fry, A.M., Nigg, E.A., and Mayer, T.U. 2005. Xenopus polo-like kinase Plx1 regulates XErp1, a novel inhibitor of APC/C activity. Genes \& Dev. 19: 502-513.

Schwab, M., Neutzner, M., Mocker, D., and Seufert, W. 2001. Yeast Hct1 recognizes the mitotic cyclin Clb2 and other substrates of the ubiquitin ligase APC. EMBO J. 20: 51655175 .

Schwickart, M., Havlis, J., Habermann, B., Bogdanova, A., Camasses, A., Oelschlaegel, T., Shevchenko, A., and Zachariae, W. 2004. Swm 1/Apc13 is an evolutionarily conserved subunit of the anaphase-promoting complex stabilizing the association of Cdc16 and Cdc27. Mol. Cell. Biol. 24: 35623576.

Searle, J.S., Schollaert, K.L., Wilkins, B.J., and Sanchez, Y. 2004. The DNA damage checkpoint and PKA pathways converge on APC substrates and Cdc20 to regulate mitotic progression. Nat. Cell Biol. 6: 138-145.

Seol, J.H., Feldman, R.M., Zachariae, W., Shevchenko, A., Correll, C.C., Lyapina, S., Chi, Y., Galova, M., Claypool, J., Sandmeyer, S., et al. 1999. Cdc53/cullin and the essential Hrt1 RING-H2 subunit of SCF define a ubiquitin ligase module that activates the E2 enzyme Cdc34. Genes \& Dev. 13: 1614 1626.

Shirayama, M., Zachariae, W., Ciosk, R., and Nasmyth, K. 1998. The Polo-like kinase Cdc5p and the WD-repeat protein Cdc20p/fizzy are regulators and substrates of the anaphase promoting complex in Saccharomyces cerevisiae. EMBO $I$. 17: 1336-1349.

Shirayama, M., Toth, A., Galova, M., and Nasmyth, K. 1999. $\mathrm{APC}(\mathrm{Cdc} 20)$ promotes exit from mitosis by destroying the anaphase inhibitor Pds1 and cyclin Clb5. Nature 402: 203207.

Skowyra, D., Craig, K.L., Tyers, M., Elledge, S.J., and Harper, J.W. 1997. F-box proteins are receptors that recruit phosphorylated substrates to the SCF ubiquitin-ligase complex. Cell 91: 209-219.

Skowyra, D., Koepp, D.M., Kamura, T., Conrad, M.N., Conaway, R.C., Conaway, J.W., Elledge, S.J., and Harper, J.W. 1999. Reconstitution of G1 cyclin ubiquitination with complexes containing SCFGrr1 and Rbx1. Science 284: 662-665.

Song, M.S., Song, S.J., Ayad, N.G., Chang, J.S., Lee, J.H., Hong, H.K., Lee, H., Choi, N., Kim, J., Kim, H., et al. 2004. The tumour suppressor RASSF1A regulates mitosis by inhibiting the APC-Cdc20 complex. Nat. Cell Biol. 6: 129-137.

Sudakin, V., Ganoth, D., Dahan, A., Heller, H., Hershko, J., Luca, F.C., Ruderman, J.V., and Hershko, A. 1995. The cyclosome, a large complex containing cyclin-selective ubiquitin ligase activity, targets cyclins for destruction at the end of mitosis. Mol. Biol. Cell 6: 185-197.

Sudakin, V., Chan, G.K., and Yen, T.J. 2001. Checkpoint inhibition of the APC/C in HeLa cells is mediated by a complex of BUBR1, BUB3, CDC20, and MAD2. J. Cell Biol. 154: 925-936.

Tang, Z., Li, B., Bharadwaj, R., Zhu, H., Ozkan, E., Hakala, K., Deisenhofer, J., and Yu, H. 2001. APC2 Cullin protein and
APC11 RING protein comprise the minimal ubiquitin ligase module of the anaphase-promoting complex. Mol. Biol. Cell 12: 3839-3851.

Tang, Z., Shu, H., Oncel, D., Chen, S., and Yu, H. 2004. Phosphorylation of Cdc20 by Bub1 provides a catalytic mechanism for APC/C inhibition by the spindle checkpoint. Mol. Cell 16: 387-397.

Thornton, B.R. and Toczyski, D.P. 2003. Securin and B-cyclin/ CDK are the only essential targets of the APC. Nat. Cell Biol. 5: 1090-1094.

Thornton, B.R., Ng, T.M., Matyskiela, M.E., Carroll, C.W., Morgan, D.O., and Toczyski, D.P. 2006. An architectural map of the anaphase-promoting complex. Genes \& Dev. 20: 449-460.

Tugendreich, S., Tomkiel, J., Earnshaw, W., and Hieter, P. 1995. $\mathrm{CDC} 27 \mathrm{Hs}$ colocalizes with $\mathrm{CDC} 16 \mathrm{Hs}$ to the centrosome and mitotic spindle and is essential for the metaphase to anaphase transition. Cell 81: 261-268.

Van Nocker, S. and Vierstra, R.D. 1991. Cloning and characterization of a $20-\mathrm{kDa}$ ubiquitin carrier protein from wheat that catalyzes multiubiquitin chain formation in vitro. Proc. Nat1. Acad. Sci. 88: 10297-10301.

Vodermaier, H.C., Gieffers, C., Maurer-Stroh, S., Eisenhaber, F., and Peters, J.M. 2003. TPR subunits of the anaphase-promoting complex mediate binding to the activator protein CDH1. Curr. Biol. 13: 1459-1468.

Wasch, R. and Cross, F.R. 2002. APC-dependent proteolysis of the mitotic cyclin Clb2 is essential for mitotic exit. Nature 418: $556-562$.

Wendt, K.S., Vodermaier, H.C., Jacob, U., Gieffers, C., Gmachl, M., Peters, J.M., Huber, R., and Sondermann, P. 2001. Crystal structure of the APC10/DOC1 subunit of the human anaphase-promoting complex. Nat. Struct. Biol. 8: 784-788.

Willems, A.R., Schwab, M., and Tyers, M. 2004. A hitchhiker's guide to the cullin ubiquitin ligases: SCF and its kin. Biochim. Biophys. Acta 1695: 133-170.

Yamano, H., Gannon, J., Mahbubani, H., and Hunt, T. 2004. Cell cycle-regulated recognition of the destruction box of cyclin B by the APC/C in Xenopus egg extracts. Mol. Cell 13: 137-147.

Yoon, H.J., Feoktistova, A., Wolfe, B.A., Jennings, J.L., Link, A.J., and Gould, K.L. 2002. Proteomics analysis identifies new components of the fission and budding yeast anaphasepromoting complexes. Curr. Biol. 12: 2048-2054.

$\mathrm{Yu}, \mathrm{H}$. 2006. Structural activation of Mad2 in the mitotic spindle checkpoint: The two-state Mad2 model versus the Mad2 template model. J. Cell Biol. 173: 153-157.

Zachariae, W., Shin, T.H., Galova, M., Obermaier, B., and Nasmyth, K. 1996. Identification of subunits of the anaphasepromoting complex of Saccharomyces cerevisiae. Science 274: 1201-1204.

Zachariae, W., Schwab, M., Nasmyth, K., and Seufert, W. 1998a. Control of cyclin ubiquitination by CDK-regulated binding of Hct1 to the anaphase promoting complex. Science 282: 1721-1724.

Zachariae, W., Shevchenko, A., Andrews, P.D., Ciosk, R., Galova, M., Stark, M.J., Mann, M., and Nasmyth, K. 1998b. Mass spectrometric analysis of the anaphase-promoting complex from yeast: Identification of a subunit related to cullins. Science 279: 1216-1219.

Zheng, N., Wang, P., Jeffrey, P.D., and Pavletich, N.P. 2000 Structure of a c-Cbl-UbcH7 complex: RING domain function in ubiquitin-protein ligases. Cell 102: 533-539.

Zheng, N., Schulman, B.A., Song, L., Miller, J.J., Jeffrey, P.D., Wang, P., Chu, C., Koepp, D.M., Elledge, S.J., Pagano, M., et al. 2002. Structure of the Cul1-Rbx1-Skp1-F boxSkp2 SCF ubiquitin ligase complex. Nature 416: 703-709. 


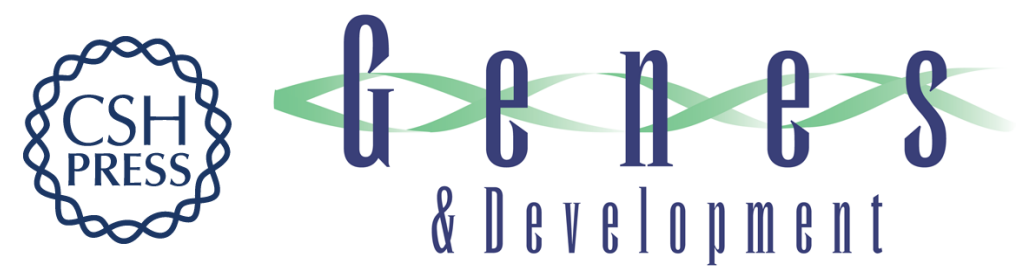

\section{Precise destruction: an emerging picture of the APC}

Brian R. Thornton and David P. Toczyski

Genes Dev. 2006, 20:

Access the most recent version at doi:10.1101/gad.1478306

References This article cites 92 articles, 39 of which can be accessed free at: http://genesdev.cshlp.org/content/20/22/3069.full.html\#ref-list-1

License

Email Alerting Receive free email alerts when new articles cite this article - sign up in the box at the top Service right corner of the article or click here.

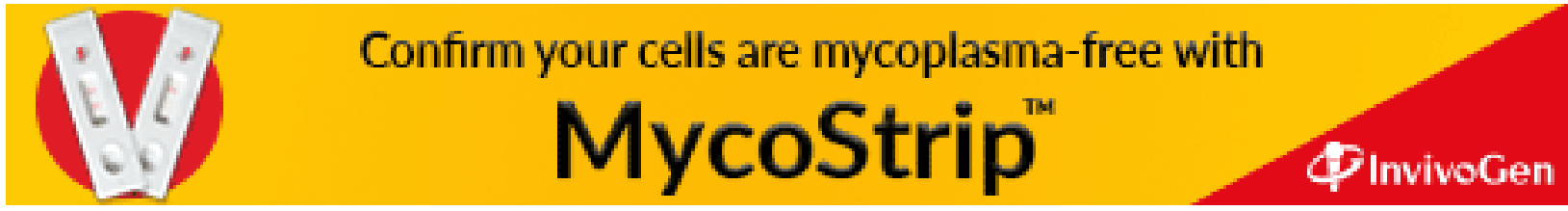

